\title{
郭正堂院士专访：评价体系改革促进中国学术期刊发展
}

刘素贞，魏建晶

\section{Improving academic evaluation system to promote Chinese scientific journals: An interview with Zhengtang Guo}

Suzhen Liu \& Jianjing Wei

doi: 10.1360/TB-2020-0613

郭正堂, 中国科学院地质与地球物理研究所研究员, 中国科学院院士, 发展中国家科学院院士. 国家自然科学 基金委员会地球科学部主任(兼), 主要从事新生代地质、古 气候学与全球变化研究. 2003 2012年担任《科学通报》执 行副主编, 2013年起任《中国科学: 地球科学》副主编.

2020年, 在《中国科学》《科学通报》(“两刊”)迎来创刊 70周年之际, 《中国科学》杂志社专访郭正堂院士, 探讨中 国学术期刊的发展及中国科技评价体系改革等问题.

\section{“两刊”促进了中国科学的传播和发展}

今年是“两刊”创办70周年，能否回忆一下您与“两 刊”之间的缘分?

郭正堂: 我与“两刊”结缘将近30年了. 1993年在《科学 通报》发表了第一篇论文. 当时, 在我国的科研评价体系 中, “两刊” 能够得到充分的认可, 因此学者们对于在“两 刊”上发表论文十分看重.

2003年, 我与郑永飞老师一起应邀担任《科学通报》 执行副主编, 负责地球科学领域稿件的处理工作. 当时 《中国科学》杂志社以 《科学通报》为“试验田”深化改革, 大力强化编委负责制, 聘用了一批像我这样的年轻科技人 员 (绝大多数是“杰青”)组建编委会. 应该说, 此举代表了 当时国内学术期刊改革的一个新起点.

郑老师对期刊工作严谨敬业而充满激情, 深深地感染 了我. 我们在《科学通报》编委会一干就是十多年, 后来 又一起进人 《中国科学: 地球科学》编委会, 一直到今天. 在这个过程中，我有机会向办刊的前辈、向同龄甚至比我 年轻的编委、向编辑、作者和审稿人都学到了很多, 与“两 刊” 结下了很深的情缘, 也深深感受到 “两刊”对我们这一 代人的成长所起到的积极作用.

70 年来, “两刊”在中国科技事业发展中扮演了怎样 的角色?

郭正堂: 我认为, “两刊”70年来在推动中国科学的发 展、学科建设和人才培养过程中都扮演了非常重要的角色.

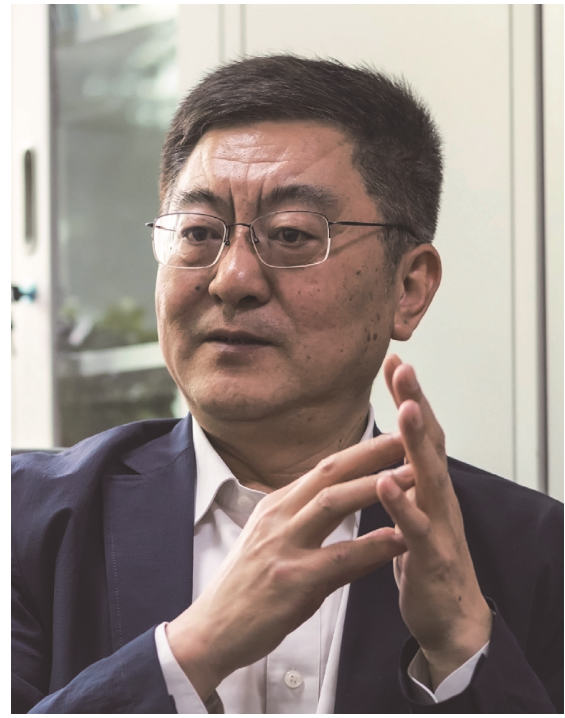

中国科学院院士 郭正堂

改革开放以前, “两刊”对促进中国科技成果的交流与 传播起到关键作用. 当时, 中国缺少学术交流的对外通道 和窗口, 而一批有影响力的成果，如牛胰岛素的人工合 成、青蒿素的结构研究、哥德巴赫猜想等相继涌现, “两刊” 促使这些原创性成果屹立于国际学术的高峰, 功不可没. 改革开放初期, 由于多方面原因, 我国学者的许多成果在 国外发表有难度，而“两刊”又承担了这个特殊时期的使命。

时至今日，虽然成果发表的途径增加了很多，但由于 学术竞争等问题, 存在一些在国外难以发表或不能快速发 表的原创性成果和思想, “两刊”对报道和传播这些成果依 然起着不可替代的作用. 另外, “两刊”在相当长的一段时 期，面对中文和外文读者群体采取的中英文版并行出版的 方式应该说是一大特色，在科学传播中起到了独特的 作用.

“两刊”的另一贡献是在人才培养中的作用. 不仅使众 多刚入门、尚不能快速准确阅读英文的学子触及到科学的 前沿，而且帮助青年科技工作者完成了从在一般国产期刊 
到“两刊”, 再到国际顶级期刊上发文的投稿过程. “两刊” 作为重要环节弥补了中间的链条, 而这个作用在人才培养 过程中是不能小视的.

\section{办好“两刊”需要长期持续的努力}

您认为“两刊”目前面临的主要问题是什么?

郭正堂: 自担任“两刊”编委起, 我就感觉到“两刊”发 展的艰难. 其中, 最主要的问题是高质量稿源偏少. 导致 这种状况的原因是多方面的, 包括我们基础研究发展的整 体水平、科研评价体系、投资强度、国际竞争环境、办刊 经验和办刊人才的积累等.

在影响“两刊”高质量稿源的各种因素中, 最直接的因 素当数科研评价体系. 自 20 世纪90年代开始, 随着中国科 研水平的提升, 越来越多的院校依据SCI评价体系进行考 评, 国外期刊在科研评价中的作用越来越重要; 另一方面, 大家也越来越希望把成果发表在能让更多学者、尤其是国 际学者看到的地方. 与此同时, “两刊”没能及时抓住契机, 实现作者群的国际化, 可能是因为我们起步较晚. 再后来, 国外期刊的数量剧增, 对稿源的竞争更加激烈, 导致“两 刊”的优质稿源大量流失. 直到现在, 国内地球科学领域 一些发展好的学科投给“两刊”的优秀稿件依然很有限, 原 因就在于许多优秀的科研成果流向了国外刊物.

高质量稿源流失是国内期刊普遍面临的问题, 对此 您对“两刊”有什么建议?

郭正堂: 导致优质稿源不足的原因是多方面的, 有办 刊本身的因素, 也有办刊以外的因素, 这是一个系统工程, 需要多方重视来逐步解决. 就近期来讲, 可以通过多种方 式“求”着作者或“逼”着作者来贡献优质稿件, 提高优质稿 件的比例和期刊的学术指标, 进而提升期刊的吸引力. 等 到我们自己的期刊有足够的吸引力, 办起来就容易多了. 这样的措施在短期内是有效的, 有些期刊已经取得了不错 的效果. 但这些方式肯定不是长久之计. 如果不改进科研 评价体系, 办刊前景是不明朗的. 更何况, 科研人员希望 将论文在读者群更大、水平更高的国际出版平台上发表, 使成果形成更大更广泛的学术影响, 也是无可非议的. 所 以, 全面办好“两刊”绝不是一䟣而就的事情, 需要较长期 的努力. 期刊本身能做的还是提高自身质量、实现办刊模 式现代化.

\section{定位、使命与学术指标是统一的整体}

高影响因子仍是当前中国SCI期刊追求的目标, 您 如何看待这个问题?

郭正堂: 目前确实有不少人把提升影响因子作为是否 成功办刊的重要指标. 重视影响因子是对的, 但也不是如 此简单. 我们在办刊过程中首先要明晰办刊的目标与定位
是什么. 如果影响因子高就算成功了, 那国际上高影响因 子的期刊已有很多，再多办一个又能如何? 高影响因子是 办刊人追求的目标之一, 但远不是全部. 办刊更多地还是 希望通过合理的定位, 完成我们赋予期刊的使命. 定位、 使命与学术指标应该是统一的整体, 且相辅相成, 不能片 面强调任何一个, 更不能对立起来. 评价一个期刊办得成 功与否, 第一要看它是否确定了自己的特色和定位, 第二 要看它是否在完成它的使命. 从这个意义上讲, 70年来的 “两刊”应当说是一个巨大的成功, 不断调整自己的定位, 出色地完成了不同时期的使命, 为促进中国科学的发展立 下汗马功劳, 在不少方面具有不可替代性, 这不是只用影 响因子就能表达的.

国外有些期刊非常强调商业化盈利，您认为“两刊” 应如何把握这个问题?

郭正堂：科技期刊出版作为一个产业, 商业盈利也是 其定位和目标中很重要的一部分. 国外也有一些学会资助 的期刊, 不是很强调商业化盈利. 对目前的国内期刊来讲, 即便希望盈利，也需要一个过程. 在中国科技期刊出版业 跻身世界强手之列以前，我们还难以且也不宜把商业盈利 作为一个主要的目标. 在现有条件下, 政府和主管部门应 该投人资金，改善办刊平台和条件，包括发行条件及人才 队伍建设等. 比如, 目前我们学术期刊编辑的水平参差不 齐. 从平均水平看, 我国编辑的英文水平还有待提高. 在 平台方面, “两刊”的平台在国内算好的, 但是与国际大出 版商相比还有一定的差距. 应该让管理人员和编辑队伍走 出去, 多看多见识, 学习世界上最先进的出版理念和经验, 进而在我们的办刊过程中发挥作用. 但不管是人才培养还 是平台建设, 最终又要回到投资的问题上. 好的工资待遇 才能吸引优秀人才, 激发人才的积极性. 我非常敬佩那些 一直坚持在科学编辑岗位上兢兢业业为国家做事的人.

\section{应将有限的资源集中用于发展 国产高水平期刊}

您刚才谈到办刊与我国基础研究发展的整体水平 有关, 可以具体谈谈吗?

郭正堂: 我国科技期刊出版业全方位国际化还需要较 长时间的努力. 在此之前, 科技期刊的水平在很大程度上 依赖于我国科技发展的整体水平，二者是分不开的. 改革 开放 40 年来, 我国科技实现了飞速发展, 应当说创造了世 界奇迹. 但我们也要意识到, 我国科技发展的整体水平与 发达国家相比还有不小的距离. 因为我国的基础科学研究 起步较晚, 积累偏少; 我国是一个发展中国家, 基础研究 的投资占研发投资的比例还远低于科技发达国家; 我国科 研人才队伍是从恢复高考后的大学生和研究生成才后才 开始较快增长的, 至今高水平人才的比例与发达国家相比 
还有一定的差距. 目前, 虽然有些领域或方向走在了国际 前沿, 但“点”不能代表“面”, “局部”不能代表“整体”. 在这 种情况下, 以我国学者为作者群主体的学术期刊也就不太 可能很快全方位超越国际高水平期刊. 所以, 看待科技期 刊的进步与看待科学发展一样, 不能过于焦躁, 太急躁了 就会出问题. 我个人认为, “两刊”对地球科学领域科研进 展的报道与中国地球科学基础研究的真实发展水平是相 适应的. 我们也应该有信心把“两刊”办好, 因为我国科技 的整体水平与世界先进水平的差距在快速缩小, 学术期刊 的水平也一定会越来越好.

对于以“两刊”为代表的中国期刊的总体状况, 您如 何评价?

郭正堂: 谈到中国期刊的总体状况, 有一句话能说明 现状, 即“我国的科技发文量位居世界第二(主要指国外期 刊上的发文量), 但原创性成果比例偏低”, 这是较普遍的 共识. 这句话至少有两重含义, 第一, 我们对国外期刊优 质稿源的贡献率有待进一步提升, 发表在国内期刊的原创 性成果比例可能更低些; 第二, 发文量相对于科学发展的 整体水平来讲有些偏高了. 虽然在发展过程中, 从数量提 升到质量提升似乎是一个必经的过程, 但目前已经到了该 强调质量的时候了, 即转型期已经来临. 在这种背景下, 应该严格控制新刊的审批, 建立相应的机制压缩刊物的数 量, 使投资更加集中. 目前, 中国科技期刊的数量非常大, 似乎已接近 5000 种, 大部分是面向国内的. 科技期刊数量 已经过多, 以至于反映原创性成果的文章比例严重降低. 我们应该集中有限的资金, 扶植包括“两刊”在内的一些优 秀国产期刊. 虽然我们的部分期刊已经发表了一些优秀稿 件, 影响因子得到了快速提升, 但在国际化和影响力方面 仍然有很大的提升空间.

最近几年开放获取(open access, OA)成为热门话题, 您对OA有什么看法?

郭正堂: $\mathrm{OA}$ 是与传统的订阅出版模式很不相同的另一 种选择. 也有人主张两种模式并行, 即订阅开放模式. OA 无疑更有利于科研成果的传播和共享, 这是 $\mathrm{OA}$ 的正面效 果. 但OA也意味着出版成本的转移和改变. 简单地讲, 传 统的订阅出版模式是 “订阅者出资”, 而OA是“发表者出 资”, 这就意味着, OA的推广会在一定程度上把科技出版 的成本从发文量小的国家向发文量大的国家转移. 由于我 国科技论文发文量已位居世界第二, 在有些国际出版集团 旗下的发文量已达到世界第一, 且在继续快速增长, OA会 增加中国科技成果出版的成本, 这一点是冊庸置疑的. 不 管是OA还是非OA, 正所谓“羊毛出在羊身上”, 最终是要 由政府出资. 从这个角度讲, OA的推广也许是一个契机, 相关机构或许可以做一个评估: 把资金用于发展我们自己 的出版平台好, 还是用于在国际成熟的出版平台上发表 OA文章以扩大我们科研成果的影响力好? 我感觉二者各
有利弊, 取决于其他各方面是否跟得上. 因为科学传播与 共享不能完全用资金来衡量, 但在实际操作中又不得不考 虑资金.

\section{改革科研评价体系，推动我国科技期刊 加快发展}

《关于深化改革培育世界一流科技期刊的意见》确 定了中国期刊的发展时间表: 到2035年, 我国科技期刊 综合实力跃居世界第一方阵. 您认为要实现这一目标, 迫切需要解决的问题是什么?

郭正堂: 有这个雄心壮志是很好的, 明确期限也是给 自己压力和动力. 发展中国的期刊事业不仅是办刊人的责 任, 也需要全社会的努力, 要把各种措施落实到实处. 第 一, 增加投资, 建设高水平的出版平台; 第二, 要培养一 批高水平的编辑出版人才. 除了这两点出版方面的举措, 还要回到评价体系的问题上. 如果不从根本上改变评价体 系, 实现这个目标还是有困难的. 现在我们还尚未实质性 地走出依据影响因子、SCI文章数量及各种各样的指标来 进行科研评价的方式. 虽然这种方式曾经为中国科学的快 速发展起到正面的作用，今天也依然有其积极的一面; 但 同时也带来了一些负面的影响，是一把“双刃剑”。我国的 科学发展到今天, 科技期刊要转型, 科技的评价体系也到 了该转型的时候了.

对于科研评价体系, 您认为目前主要的问题是什 么?

郭正堂：目前科技评价的核心在于如何处理好发文的 数量和质量的平衡. 数量和质量本来应该是一个统一的整 体, 二者并不对立. 好的科研成果如果不涉密, 一定要发 表出来, 如果发表数量和质量都很理想, 那当然非常好. 但人的时间和精力都是有限的，兼顾并不容易. 目前, 到 了该强调科研成果原创性的时候了. 科学研究的最终影响 力在于对人类认知的贡献. 我国今天如此庞大的科研队 伍, 如果平均每人在一生中能做一件有较好原创性的工 作，我们在世界科学界的地位就会大幅度提升.

评价体系中对“计数”(包括各种数字)给予的权重太大, 已不利于提高我国基础研究的原创性，不利于引导科研人 员做大事、做难事, 从而导致较多的重复性研究, 也会促 使过多的人力、物力和财力聚集在一些“短平快”的方向上. 长此下去, 有可能引发一些原创性并不高的“研究热点”, 甚至增加引用率中与质量和真正学术影响关系不大的“群 体体量效应”. “计数”权重过大甚至会助长成果的拆分发 表, 也不利于引导年轻科研人员在一个方向上做深做精, 成长为所在领域真正的行家. 有些问题实际上已有显现, 值得引起重视.

当然, 评价体系也还有其他方面需要完善. 比如, 只重 
视科研成果发表情况, 评价内容显得过于单一化, 导致部 分科研人员不愿做科学公益性工作, 不愿在学科建设、平台 建设和人才培养上花精力, 也是常见的; 而这些工作对于 国家的科技事业、对一个单位和集体的发展却是非常重要 的. 又比如, 过多地强调第一作者论文, 可能会助长科研人 员“单打独斗”及“小作坊”式科研, 不太有利于科研合作.

您对评价体系的改进有什么建议?

郭正堂: 在这个方面, 自2018年国家对项目评审和人 才评价提出“代表作评价制度”后, 中国科学院、教育部和科 技部等部门都明确要建立科学的评价体系, 注重成果质量 与创新点, 这会引导科研人员从重视数量向重视质量转变. 国家自然科学基金委员会地球科学部在人才项目评审中除 了强调代表作评价制度外, 还使用4条标准: (1) 方法学创 新: 是否创立了原创性的科学研究方法, 可被用来解决重 要的科学问题? (2) 关键科学证据: 是否为重要科学问题的 解决提供了新的、关键的、可靠的证据? (3) 理论认知/社会 需求: 是否对所在学科的认知体系或对解决重要社会需求 背后的基础科学问题有实质贡献? (4) 学科发展: 研究工作 是否可导致领域研究方向、范畴、视野/视角的变革或领域 认知体系的整体进步, 从而促进学科发展? 学者在介绍发 表论文情况的同时, 更要说明成果在这4条中能占到哪条 或哪几条, 供评审专家来推敲. 目前看来, 一项成果, 只要 能占 4 条中的 1 条, 就算是成功的. 这些标准是经过较广泛 调研制定的, 具有较好的可操作性, 可用来评价基础研究
的原创性. 如果能够按照这样的思路来评价科研人员的贡 献，作者在发表研究成果时就不会刻意区分国内和国外刊 物, 对“两刊”的发展也会起到非常好的促进作用.

您几次强调人才队伍建设, 能否进一步谈谈, 特别 是评价体系与人才队伍建设的关系?

郭正堂: 无论是科学本身的发展还是学术期刊的发 展, 人才的积累都是第一位的. 大到一个国家、部门或单 位, 小到一个课题组, 谁拥有更多的高水平科研人才, 谁 的科研水平一般就相对高. 根据一个部门或单位目前的青 年科技队伍的状况, 不难看出 10 年后谁会拥有最优秀的队 伍，也就不难看出其发展前景.

就科技评价体系本身的问题来讲，肯定有政策方面的 原因, 但从根子上还有一个人才队伍的积累问题，需要一 个过程才能彻底改变. 这些年来, 虽然我国高水平科技人 才的数量快速增加, 涌现出不少非常优秀的科学家, 但这 个数量与科技发达国家相比还是偏少, 还不能满足需求. 换句话说, 高学术鉴赏力的科学家群体的体量偏小, 也是 “计数式”评价方式得以盛行的原因之一. 道理很简单, 你让 我评价一个成果, 但我不太懂, 我就只好“计数”. 另外, 我 们各种各样的“评比”也显然太多了些, 更加剧了这种状况.

作为科技期刊编辑, 我们深知广大科研人员对中国 的科技期刊给予了很高的期望. 我们会一如既往地带 着崇高的使命感去努力办好“两刊”, 不负大家的期待, 为提高我国科技期刊的综合实力作出贡献. 